\title{
Esophageal Cancer Surgery: Spontaneous Centralization in the US Contributed to Reduce Mortality Without Causing Health Disparities
}

\author{
Francisco Schlottmann, MD ${ }^{1,2}$, Paula D. Strassle, MSPH ${ }^{1,3}$, Anthony G. Charles, MD ${ }^{1}$, and Marco G. Patti, MD ${ }^{1,4}$ \\ ${ }^{1}$ Department of Surgery, University of North Carolina at Chapel Hill, Chapel Hill, NC ; ${ }^{2}$ Department of Surgery, Hospital \\ Alemán of Buenos Aires, Buenos Aires, Argentina; ${ }^{3}$ Department of Epidemiology, Gillings School of Global Public \\ Health, University of North Carolina at Chapel Hill, Chapel Hill, NC; ${ }^{4}$ Department of Medicine, University of North \\ Carolina, Chapel Hill, NC
}

\begin{abstract}
Background. Improvement in mortality has been shown for esophagectomies performed at high-volume centers.

Objective. This study aimed to determine if centralization of esophageal cancer surgery occurred in the US, and to establish its impact on postoperative mortality. In addition, we aimed to analyze the relationship between regionalization of cancer care and health disparities.

Methods. A retrospective population-based analysis was performed using the National Inpatient Sample for the period 2000-2014. Adult patients ( $\geq 18$ years of age) diagnosed with esophageal cancer and who underwent esophagectomy were included. Yearly hospital volume was categorized as low $(<5$ procedures $)$, intermediate $(5-20$ procedures), and high ( $>20$ procedures). Multivariable analyses on the potential effect of hospital volume on patient outcomes were performed, and the yearly rate of esophagectomies was estimated using Poisson regression.

Results. A total of 5235 patients were included. Esophagectomy at low- [odds ratio (OR) 2.17] and intermediate-volume (OR 1.62) hospitals, compared with highvolume hospitals, was associated with a significant increase in mortality. The percentage of esophagectomies performed at high-volume centers significantly increased during the study period $(29.2-68.5 \% ; p<0.0001)$. The trend towards high-volume hospitals was different among the different US regions: South (7.7-54.3\%), West
\end{abstract}

First Received: 19 September 2017;

Published Online: 18 January 2018

F. Schlottmann, MD

e-mail: fschlottmann@hotmail.com
(15.0-67.6\%), Midwest (37.3-67.7\%), and Northeast $(55.8-86.8 \%)[p<0.0001]$. Overall, the mortality rate of esophagectomy dropped from 10.0 to $3.5 \%(p=0.006)$, with non-White race, public insurance, and low household income patients also showing a significant reduction in mortality.

Conclusions. A spontaneous centralization for esophageal cancer surgery occurred in the US. This process was associated with a decrease in the mortality rate, without contributing to health disparities.

Keywords Esophageal cancer - Centralization · Mortality · Disparities

The incidence of esophageal cancer, particularly esophageal adenocarcinoma, is expected to rise dramatically in many Western countries. ${ }^{1}$ Surgical resection is the cornerstone of curative treatment. Although there has been a significant improvement in operative techniques and postoperative care, esophagectomy remains one of the most demanding surgical procedures, with significant associated morbidity and mortality. ${ }^{2,3}$

The relationship between hospital operative volume and postoperative mortality rates after complex surgical procedures has been clearly established. ${ }^{4-7}$ Specifically, it has been shown that operative volume is an important determinant of quality of care for esophagectomy; ${ }^{8-11}$ thus, the potential advantages of centralizing esophageal cancer surgery continue to be discussed in many healthcare systems. With the lack of uniform prescriptive guidelines or operative volume standards implementation, the attainment of centralization of esophageal cancer surgery is currently still aspirational in the US, which can be attributed to several factors. First, many patients prefer to seek 
definitive cancer care near home at their local community hospital rather than travel far to an unknown center. Second, with variations in healthcare system networks across the country, determining centers of excellence designation and steering patient referrals to such centers is challenging. Lastly, the financial implications of patient referral to highvolume centers may be a disincentive to centralization of care.

Scarce data are available regarding the occurrence of a nationwide spontaneous concentration of esophageal cancer surgery in high-volume centers. We therefore aimed to characterize the trend of centralization of esophageal cancer surgery in the US and to determine its impact on postoperative mortality. In addition, we aimed to analyze the relationship between regionalization of cancer care and health disparities.

\section{METHODS}

We performed a retrospective analysis of the National Inpatient Sample (NIS) database between 1 January 2000 and 31 December 2014. The NIS is the largest publically available all-payer healthcare database in the US, and includes over 7 million hospitalizations from 1000 hospitals each year, representing a $20 \%$ stratified sample of all hospital discharges in the US. Eligible patients were identified using International Classification of Disease, 9th Revision, Clinical Modification (ICD-9-CM) diagnostic and procedural codes.

Adult patients ( $\geq 18$ years of age) diagnosed with esophageal cancer (ICD-9-CM 150-150.9) who underwent an elective esophagectomy (42.4-42.42, 42.58, and 42.6-42.69) during their inpatient hospitalization were eligible for inclusion. Yearly hospital volume was calculated by summing the number of patients and applying the discharge weights included in the NIS, which weights observations so that counts are nationally representative. Patients with missing weights or weights of 0 were excluded $(n=8)$.

Surgical outcomes of interest were inpatient mortality, postoperative complications during the index hospitalization, hospital length of stay, and total charges (excluding operating room time costs). Postoperative complications included venous thromboembolism (415.11, 453.40-453.42, and V12.51), wound complications (998.13, 998.30-998.32, and 998.83), infection (54.91, 86.04, 567.22, 569.5, 995.9-995.99, 996.64, 998.5-998.59, and 999.3-999.39), esophageal perforation (42.82 and 530.4), postoperative bleeding (99.0-99.09, 998.11, and 998.12), shock (998.0-998.09), cardiac failure (410-410.9, 428-428.9), renal failure $(38.95,39.95,584-584.9,586$, and V45.11), and respiratory failure (31.1-31.29, 96.04,
96.05, 96.7-96.72, and 799.1). A composite complication (i.e. at least one postoperative complication) was also analyzed.

Comorbidities of interest included hypertension (401-401.9 and 402-402.91), primary and secondary diabetes (249-249.91 and 250-250.93), obesity (278-278.8), renal insufficiency (585-585.9), coronary artery disease (414-414.9), peripheral vascular disease (443-443.9), chronic obstructive pulmonary disease (491-492.8), and sleep apnea (327.23).

\section{Statistical Analysis}

Yearly hospital volume was categorized as low $(<5$ procedures), intermediate (5-20 procedures), and high (> 20 procedures). Patient demographics, hospital characteristics, and procedure type were compared across hospital surgical volume using Chi square $\left(X^{2}\right)$ and analysis of variance (ANOVA) tests, where appropriate. Unadjusted, bivariate analyses of inpatient mortality, length of stay, hospital charges, and complication incidence across hospital surgical volume were conducted using Chi square $\left(X^{2}\right)$ and Wilcoxon-Mann-Whitney tests.

Missing data for race/ethnicity $(n=1004,19.2 \%)$, primary insurance $(n=20,0.4 \%)$, household income $(n=124,2.4 \%)$, hospital teaching status $(n=9,0.2 \%)$, bed size $(n=9,0.2 \%)$, inpatient mortality $(n=4,0.1 \%)$, and hospital charges $(n=171,3.3 \%)$ were estimated using Markov Chain Monte Carlo (MCMC) multiple imputation $(n=40)$. A non-informative prior, 200 burn-in iterations and 100 iterations between imputations was specified. MCMC models included the variables with missing data plus all postoperative complications, length of stay, admit year, age, comorbidities, and hospital region. Variable estimates were not rounded or bounded.

Multivariable analyses on the potential effect of hospital volume on patient outcomes were performed on the imputed datasets using linear and logistic regression, where appropriate. Models were adjusted for admit year, sex, age, race/ethnicity, comorbidities, primary insurance, household income, hospital region, hospital size, and teaching status. Age was modeled as a restricted cubic spline.

The yearly rate of esophagectomies, stratified by hospital volume category, was estimated using Poisson regression. The yearly rate of esophagectomies at highvolume centers, stratified by US Census regions (Northeast, Midwest, South, and West), was also estimated using Poisson regression. ${ }^{12}$ Differences across regions were assessed using a likelihood ratio test (LRT). Due to changes in NIS sampling strategy, discharge records from 2012 to 2014 were excluded in all trend analyses. Additionally, the yearly rate of postoperative mortality, stratified by race (non-Hispanic White vs. other race), household income 
(lowest vs. medium/high/highest), and primary insurance type (private vs. public) was assessed using Poisson regression, and differences across groups were assessed using LRT.

All analyses were performed using SAS software version 9.4 (SAS Institute, Inc., Cary, NC, USA). A $p$ value $<0.05$ was considered significant.

\section{RESULTS}

A total of 5235 patients were included. During the study period, $52.2 \%$ of patients underwent esophagectomy in high-volume hospitals, $35.0 \%$ in intermediate-volume hospitals, and $12.8 \%$ in low-volume hospitals. Non-Hispanic White race, private primary insurance, and higher household income were more prevalent at high-volume hospitals $(p<0.0001)$. The majority $(94.8 \%)$ of the highvolume centers consisted of urban teaching hospitals $(p<0.0001)$. Patient and hospital characteristics, stratified by hospital volume, are described in Table 1 .

Compared with high-volume hospitals, low- and intermediate-volume hospitals had a significantly higher incidence of postoperative infection (16.5 and $14.4 \%$, respectively, vs. $12.0 \% ; p=0.003)$, bleeding (24.9 and $20.2 \%$ vs. $18.8 \% ; p=0.003)$, cardiac failure $(7.6$ and $6.4 \%$ vs. $5.1 \% ; p=0.02)$, renal failure $(10.0$ and $8.5 \%$ vs. $6.4 \% ; p=0.001)$, respiratory failure ( 28.3 and $24.4 \%$ vs. $18.5 \% ; p<0.0001)$, and inpatient mortality $(10.2$ and $6 / 7 \%$ vs. $3.9 \% ; p<0.0001)$. The median length of hospital stay was 14 days (interquartile range [IQR] 10-20) for low-volume hospitals, 12 days (IQR 9-19) for intermediate-volume hospitals, and 11 days (IQR 8-16) for highvolume hospitals $(p<0.0001)$. Before adjustment, no significant differences were seen in the incidence of wound complications $\quad(p=0.69)$, esophageal perforation ( $p=0.39)$, or median hospital charges $(p=0.19)$.

After adjusting for patient and hospital characteristics, patients at low-volume hospitals were significantly more likely to have a complication [odds ratio (OR) 1.40, 95\% confidence interval (CI) $1.15-1.70 ; p=0.0007]$, whereas no significant difference in the overall incidence of complications was seen in intermediate hospitals (OR 1.10, 95\% CI $0.97-1.26 ; p=0.14$ ) [Table 2]. Specifically, patients at low-volume hospitals were more likely to have postoperative infection (OR 1.52, 95\% CI 1.16-2.00), bleeding (OR 1.36, 95\% CI 1.08-1.72), renal failure (OR $1.74,95 \%$ CI $1.23-2.47$ ), respiratory failure (OR $1.58,95 \%$ CI 1.26-1.98), and inpatient mortality (OR 2.17, 95\% CI 1.49-3.15). While overall complications were not different, patients at intermediate-volume hospitals were more likely to have postoperative infection (OR 1.25, 95\% CI 1.03-1.52), renal failure (OR 1.34, 95\% CI 1.04-1.73), respiratory failure (OR 1.36, 95\% CI 1.15-1.60), and inpatient mortality (OR 1.62, 95\% CI 1.20-2.17). On average, patients at low-volume hospitals stayed 1.74 days longer (95\% CI $0.32-3.15$ ) and patients at intermediatevolume hospitals stayed 1.48 days longer $(95 \%$ CI 0.51-2.45).

Between 2000 and 2011, the rate of procedures across hospital volume significantly changed in the US. Specifically, the percentage of esophagectomies performed at high-volume centers increased from 29.2 to $68.5 \%$, while the percentage at low- and intermediate-volume hospitals decreased from 24.9 to $9.6 \%$ and 45.9 to $21.9 \%$, respectively $(p<0.0001)$ [Fig. 1]. The trend towards highvolume hospitals was different among the different country regions: South (7.7-54.3\%), West (15.0-67.6\%), Midwest $(37.3-67.7 \%)$, and Northeast $(55.8-86.8 \%)(p<0.0001)$ [Fig. 2].

Overall, between 2000 and 2011, the inpatient mortality rate after esophagectomy dropped from 10.0 to $3.5 \%$ $(p=0.006)$. When stratified by household income, the average reduction in yearly mortality was significantly higher among low household income patients (30.0-2.3\%) than medium/high/highest household income patients $(9.1-3.6 \%)[p=0.02]$. While the rates of mortality were different between non-Hispanic White patients and other race patients in 2000 ( $8.5 \%$ vs. $21.1 \% ; p<0.0001)$, the average decrease in mortality over time was relatively consistent between the two groups $(p=0.13)$. Similarly, although the rates of mortality were significantly different in 2000 between public and private primary insurance patients $(14.3 \%$ vs. $3.9 \% ; p<0.0001)$, there were similar decreases in mortality across the two groups $(p=0.10)$ [Fig. 3].

\section{DISCUSSION}

The aims of this study were to determine if a process of spontaneous centralization of esophageal cancer surgery occurred in the US, and to establish its impact on postoperative mortality. We found that the percentage of procedures performed at high-volume hospitals significantly increased nationwide in the last decade, and the postoperative mortality rate dropped from $10.0 \%$ in 2000 to $3.5 \%$ in 2011.

Several studies have shown the benefits of concentrating esophageal cancer surgery in high-volume centers. ${ }^{8-11}$ Wouters et al. ${ }^{8}$ analyzed a cohort of patients who underwent esophagectomy after a centralization project in The Netherlands. They found that along with a reduction in postoperative morbidity and length of stay, mortality fell from 12 to $4 \% .^{8}$ Markar et al. ${ }^{9}$ performed a meta-analysis and demonstrated an increase in 30-day mortality and 
TABLE 1 Distribution of patient and hospital characteristics among adult patients undergoing esophagectomy between 2000 and 2014 $(n=5235)$

Low volume [671 (12.8\%)] Intermediate volume [1831 (35.0\%)] High volume [2733 (52.2\%)] $p$ value

$$
\text { Sex }
$$

Male

Female

Age, years [mean (SD)]

Race/ethnicity

Non-hispanic white

Non-hispanic black

Hispanic

Other

Missing

Primary insurance

Private
Public
Other/self-pay

Other/self-pay

Low
Medium
High
Highest
Comorbidities
Hypertension
Diabetes
Obesity
Renal insufficiency
Coronary artery disease
Peripheral vascular disease
COPD

Sleep apnea

Hospital size

$\begin{array}{ll}\text { Small } & 69(10.3) \\ \text { Medium } & 191(28.6) \\ \text { Large } & 407(61.0) \\ \text { Hospital type } & \\ \text { Urban, teaching } & 349(52.3) \\ \text { Urban, non-teaching } & 277(41.5) \\ \text { Rural, non-teaching } & 41(6.2) \\ \text { Hospital region } & \\ \text { Northeast } & 90(13.4) \\ \text { Midwest } & 178(26.5) \\ \text { South } & 252(37.6) \\ \text { West } & 151(22.5)\end{array}$

274 (41.1)

363 (54.4)

30 (4.5)

117 (17.9)

181 (27.8)

186 (28.5)

168 (25.8)

$242(36.1)$

109 (16.2)

37 (5.5)

23 (3.4)

84 (12.5)

12 (1.8)

23 (3.4)

21 (3.1)

151 (22.5)

$1468(80.2)$
$363(19.8)$
$63.5(10.0)$
$1269(85.5)$
$97(6.5)$
$57(3.8)$
$61(4.1)$
347

$$
\begin{array}{r}
723(39.6) \\
1030(56.4) \\
72(4.0)
\end{array}
$$

341 (19.0)

448 (24.9)

506 (28.2)

502 (27.9)

804 (43.9)

313 (17.1)

118 (6.4)

59 (3.2)

228 (12.5)

42 (2.3)

83 (4.5)

85 (4.6)

134 (7.3)

360 (19.7)

1332 (73.0)

1363 (74.6)

395 (21.6)

68 (3.7)

323 (17.6)

\begin{tabular}{|c|c|}
\hline $2240(82.0)$ & 0.27 \\
\hline 493 (18.0) & - \\
\hline $63.4(10.3)$ & 0.81 \\
\hline 1999 (90.1) & $<\mathbf{0 . 0 0 0 1}$ \\
\hline $75(3.4)$ & $<\mathbf{0 . 0 0 0 1}$ \\
\hline $81(3.7)$ & 0.75 \\
\hline $63(2.8)$ & 0.10 \\
\hline 515 & - \\
\hline $1315(48.3)$ & $<0.0001$ \\
\hline 1319 (48.4) & $<\mathbf{0 . 0 0 0 1}$ \\
\hline $89(3.3)$ & 0.23 \\
\hline 492 (18.5) & 0.83 \\
\hline 629 (23.6) & 0.08 \\
\hline 715 (26.9) & 0.53 \\
\hline $826(31.0)$ & 0.009 \\
\hline 1217 (44.5) & 0.0003 \\
\hline 418 (15.3) & 0.26 \\
\hline $155(5.7)$ & 0.50 \\
\hline $52(1.9)$ & 0.007 \\
\hline $386(14.1)$ & 0.21 \\
\hline $64(2.3)$ & 0.68 \\
\hline 79 (2.9) & 0.01 \\
\hline 98 (3.6) & 0.11 \\
\hline $206(7.5)$ & 0.03 \\
\hline 303 (11.1) & $<0.0001$ \\
\hline $2224(81.4)$ & $<0.0001$ \\
\hline $2590(94.8)$ & $<0.0001$ \\
\hline 77 (2.8) & $<\mathbf{0 . 0 0 0 1}$ \\
\hline $66(2.4)$ & $<\mathbf{0 . 0 0 0 1}$ \\
\hline $862(31.5)$ & $<\mathbf{0 . 0 0 0 1}$ \\
\hline 619 (22.7) & 0.03 \\
\hline 722 (26.4) & $<\mathbf{0 . 0 0 0 1}$ \\
\hline 530 (19.4) & 0.15 \\
\hline
\end{tabular}

464 (25.3)

661 (36.1)

383 (20.9)

Data are expressed as $n(\%)$ unless otherwise specified Bold values indicate statistical significance $(p<0.05)$

$S D$ standard deviation, $C O P D$ chronic obstructive pulmonary disease

${ }^{a}$ Between 2000 and 2002, household income was characterized by the following quartiles: \$1-\$24,999 (low), \$25,000-\$34,999 (medium), $\$ 35,000-\$ 44,999$ (high), and $\$ 45,000$ and above (highest); from 2003 onward, income was characterized into quartiles within each ZIP code 
TABLE 2 Adjusted odds ratios of low and intermediate surgical-volume hospitals, compared with high-volume hospitals, on postoperative complications, length of stay, and hospital charges among adult patients undergoing esophagectomy

\begin{tabular}{|c|c|c|c|c|c|c|c|}
\hline & & \multicolumn{3}{|c|}{ Low volume } & \multicolumn{3}{|c|}{ Intermediate volume } \\
\hline & & $\mathrm{OR}^{\mathrm{a}}$ & $95 \% \mathrm{CI}$ & $p$ value & $\mathrm{OR}^{\mathrm{a}}$ & $95 \% \mathrm{CI}$ & $p$ value \\
\hline \multicolumn{8}{|l|}{ Postoperative complications } \\
\hline Venous thromboembolism & & 0.67 & $0.44-1.02$ & 0.06 & 0.72 & $0.55-0.95$ & 0.02 \\
\hline Wound complications & & 0.79 & $0.43-1.45$ & 0.44 & 1.01 & $0.69-1.48$ & 0.95 \\
\hline Infection & & 1.52 & $1.16-2.00$ & 0.002 & 1.25 & $1.03-1.52$ & 0.03 \\
\hline Esophageal perforation & & 1.70 & $0.54-5.32$ & 0.36 & 1.82 & $0.84-3.94$ & 0.13 \\
\hline Bleeding & & 1.36 & $1.08-.72$ & 0.008 & 1.09 & $0.93-1.29$ & 0.29 \\
\hline Cardiac failure & & 1.42 & $0.96-2.09$ & 0.08 & 1.25 & $0.94-1.66$ & 0.12 \\
\hline Renal failure & & 1.74 & $1.23-2.47$ & 0.002 & 1.34 & $1.04-1.73$ & 0.02 \\
\hline Respiratory failure & & 1.58 & $1.26-1.98$ & $<\mathbf{0 . 0 0 0 1}$ & 1.36 & $1.15-1.60$ & 0.0002 \\
\hline Shock & & 2.03 & $0.83-5.00$ & 0.12 & 1.85 & $0.93-3.67$ & 0.08 \\
\hline Mortality & & 2.17 & $1.49-3.15$ & $<\mathbf{0 . 0 0 0 1}$ & 1.62 & $1.20-2.17$ & 0.002 \\
\hline \multirow[t]{2}{*}{ Any complication ${ }^{\mathrm{b}}$} & & 1.40 & $1.15-1.70$ & 0.0007 & 1.10 & $0.97-1.26$ & 0.14 \\
\hline & CIE & & $95 \% \mathrm{CI}$ & $p$ value & CIE & $95 \% \mathrm{CI}$ & $p$ value \\
\hline Length of stay, days & 1.74 & & $0.32-3.15$ & 0.02 & 1.48 & $0.51,2.45$ & 0.003 \\
\hline Charges, thousands & -5.04 & & $-23.23-13.16$ & 0.59 & -7.96 & $-20.40-4.48$ & 0.21 \\
\hline
\end{tabular}

Bold values indicate statistical significance $(p<0.05)$

$O R$ odds ratio, $C I$ confidence interval, $C I E$ change in estimate

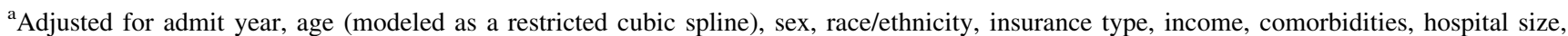
location/teaching status, and region; missing data were imputed using Markov Chain Monte Carlo multiple imputation

${ }^{\mathrm{b}}$ At least one postoperative complication (compared with no complications)

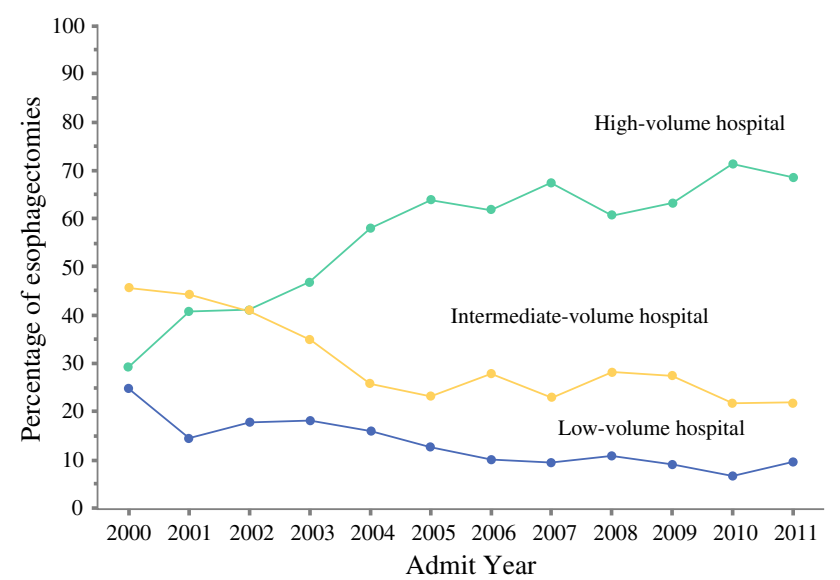

FIG. 1 Yearly rate of esophagectomies in the US, stratified by hospital volume

inhospital mortality associated with esophagectomy performed at low-volume hospitals. Similarly, a recent European multicenter study showed that low-volume centers were associated with increased 30-day postoperatively mortality. ${ }^{11}$ However, different thresholds have been used to define low- and high-volume institutions. For instance, The American Leapfrog group established a minimum hospital case volume of 13 esophageal resections in response to known improved outcomes in larger-volume centers. ${ }^{13}$ We decided to adopt a cut-off of 20 operations per year for high-volume hospitals, based on previous studies, to achieve low postoperative mortality. ${ }^{14,15} \mathrm{We}$ further classified into low ( $<5$ procedures) and intermediate (5-20 procedures) to differentiate centers with very few cases per year. In our analysis, both patients at low(OR 2.17) and intermediate-volume centers (OR 1.62) had significantly higher incidences of postoperative mortality compared with high-volume centers. In addition, highvolume hospitals were associated with less postoperative morbidity and shorter length of hospital stay. Our data suggest that the higher mortality in low- and intermediatevolume hospitals was probably a consequence of the higher morbidity seen at these centers, and a lower ability to rescue. In addition, other factors across hospitals played a role, since operative mortality rates are unlikely to be a linear product of any single factor, such as volume. Improvements in surgical technique and perioperative care, dedicated anesthetic teams, and high dependency units certainly contributed. In addition, the multidisciplinary approach for esophageal cancer management at specialized centers determines a better patient selection. 

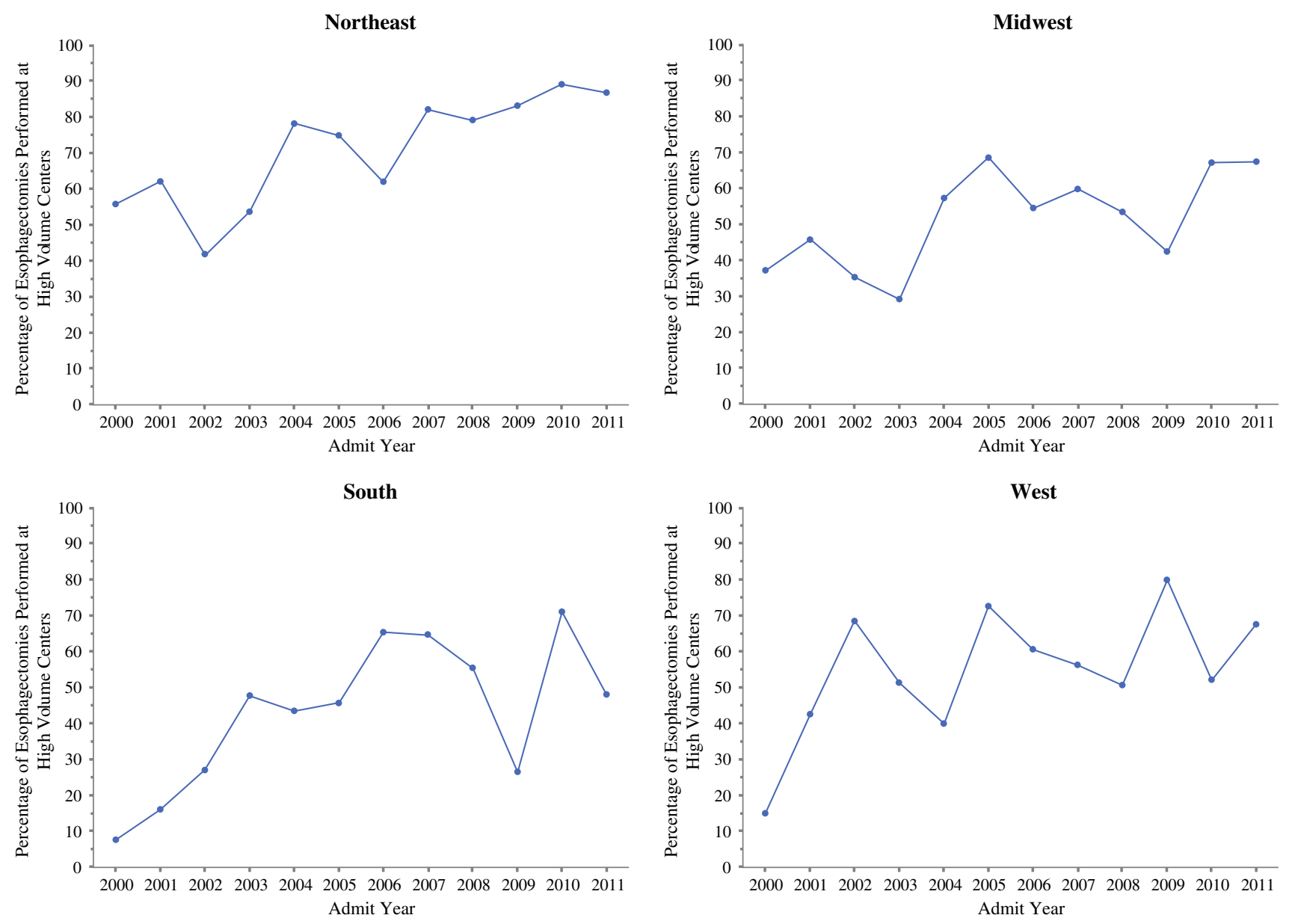

FIG. 2 Yearly rate of esophagectomies performed at high-volume centers, stratified by US regions

Interestingly, despite numerous obstacles, the US has experienced a spontaneous centralization of esophageal cancer surgery towards high-volume centers in the last decade. While in 2000 the percentage of esophagectomies performed at high-volume centers was $29.2 \%$, this number rose to $68.5 \%$ in 2011. This trend demonstrates how reporting volume-outcomes data drives patients and professional practice. Without restrictions and without designated centers of excellence, esophageal cancer patients have flowed towards high-volume hospitals. This shift might be due in part to the process of consolidation of healthcare systems that has occurred in the last decade. Small community hospitals have joined large academic centers so that the more complex procedures are performed in high-volume centers. In addition, the medical board of these new healthcare systems might not grant privileges for operations such as esophagectomies to surgeons who previously performed one or two of these procedures per year. Finally, as the data on the relationship between volume and outcome are today of public dominion, individual surgeons might be more reluctant to perform operations linked to high morbidity and mortality for the fear of litigation. ${ }^{16}$
As we intended to capture a broad snapshot of the cancer care delivery system in the entire US, we also analyzed the trend of centralization in the different regions of the country. Remarkably, in 2000 only $7 \%$ of the esophagectomies were performed at high-volume hospitals in the South. While this percentage increased to $54.3 \%$ by 2011, we believe this number should be higher. Compared with the South, the Northeast had a baseline of $55.8 \%$ procedures at high-volume centers in 2000 , increasing to $86.8 \%$ in 2011. These findings might be explained by the high concentration of tertiary and quaternary hospitals in a relatively small region, which allow patients to travel shorter distances. We can also speculate that higher socioeconomic status allowed more patients and their families to travel to urban teaching hospitals. Overall, we believe geographical and socioeconomic barriers for access to high-quality cancer care should be explored.

Along with the centralization of esophageal cancer surgery in the US, the overall mortality rate after esophagectomy dropped from $10.0 \%$ in 2000 to $3.5 \%$ in 2011. As we were concerned that regionalization of cancer care could contribute to health disparities, we analyzed 

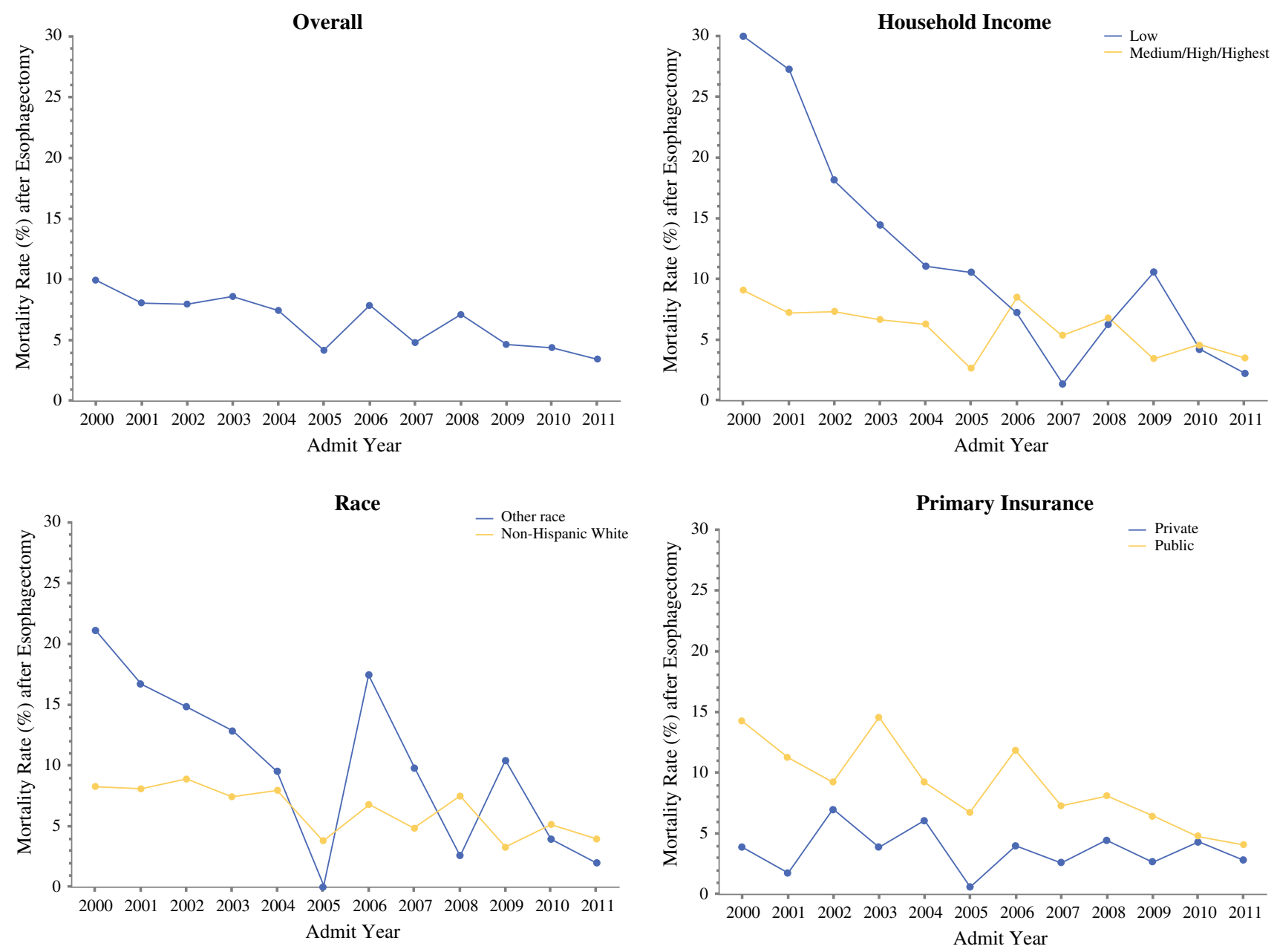

FIG. 3 Yearly rate of inpatient mortality after esophagectomy in the US for the overall population, stratified by race, household income level, and insurance type

whether vulnerable populations would also benefit by this process. Interestingly, the reduction in postoperative mortality was higher among low household income patients, and showed no significant differences between non-Hispanic White patients and other race patients, as well as between private primary insurance patients and public primary insurance patients. A further centralized network could be challenging because patients would need to travel longer distances to undergo surgery in a regional center of excellence. A recent study reported that esophageal cancer patients who travel longer distances to high-volume centers have significantly different treatment and better outcomes than patients who stay close to home at low-volume centers. ${ }^{17}$ Therefore, healthcare providers and payers should be encouraged to address the economic impact of a centralized cancer care system in order to avoid disparities in access to care at a population level. Overall, our findings suggest that centralization of esophageal cancer care did not result in impaired access to care.
Limitations of our study include that the NIS does not link hospital records, meaning that patient outcomes, including complications, re-admission, and mortality, occurring after the initial hospital discharge were unable to be measured. There is also potential for coding errors and differences in coding practices across hospitals in a large administrative database. In addition, the NIS dataset is limited by the lack of cancer-specific information, such as stage, cell type, or the utilization of neoadjuvant therapy. Finally, we did not make any distinction between different surgical approaches and reconstructive techniques.

Despite these limitations, our study shows the benefits of concentrating esophageal cancer surgery in high-volume centers, and the temporal trend of centralization of esophagectomies for cancer across the US.

\section{CONCLUSIONS}

The US experienced a nationwide spontaneous centralization towards high-volume centers for the surgical 
treatment of esophageal cancer. This process contributed to reducing the mortality rate after esophagectomy without causing health disparities.

DISCLOSURE Francisco Schlottmann, Paula D. Strassle, Anthony G. Charles, and Marco G. Patti have no conflicts of interest to declare.

\section{REFERENCES}

1. Arnold M, Laversanne M, Brown LM, et al. Predicting the future burden of esophageal cancer by histological subtype: international trends in incidence up to 2030. Am J Gastroenterol. 2017;112(8):1247-1255.

2. Sauvanet A, Mariette C, Thomas P, et al. Mortality and morbidity after resection for adenocarcinoma of the gastroesophageal junction: predictive factors. J Am Coll Surg. 2005;201:253-262.

3. Kassis ES, Kosinski AS, Ross P Jr, et al. Predictors of anastomotic leak after esophagectomy: an analysis of the society of thoracic surgeons general thoracic database. Ann Thorac Surg. 2013;96(6):1919-1926.

4. Birkmeyer JD, Siewers AE, Finlayson EV, et al. Hospital volume and surgical mortality in the United States. $N$ Engl J Med. 2002;346(15):1128-1137.

5. Gasper WJ, Glidden DV, Jin C, et al. Has recognition of the relationship between mortality rates and hospital volume for major cancer surgery in California made a difference? A followup analysis of another decade. Ann Surg. 2009;250(3):472-483.

6. Finks JF, Osborne NH, Birkmeyer JD. Trends in hospital volume and operative mortality for high-risk surgery. $N$ Engl $J$ Med. 2011;364(22):2128-2137.

7. Reames BN, Ghaferi AA, Birkmeyer JD, et al. Hospital volume and operative mortality in the modern era. Ann Surg. 2014;260(2): 244-251.
8. Wouters MW, Karim-Kos HE, le Cessie S, et al. Centralization of esophageal cancer surgery: does it improve clinical outcome? Ann Surg Oncol. 2009;16(7):1789-1798.

9. Markar SR, Karthikesalingam A, Thrumurthy S, et al. Volumeoutcome relationship in surgery for esophageal malignancy: systematic review and meta-analysis 2000-2011. J Gastrointest Surg. 2012;16(5):1055-1063.

10. Henneman D, Dikken JL, Putter $\mathrm{H}$, et al. Centralization of esophagectomy: how far should we go? Ann Surg Oncol. 2014;21(13):4068-4074.

11. Markar S, Gronnier C, Duhamel A, et al. Pattern of postoperative mortality after esophageal cancer resection according to center volume: results from a large european multicenter study. Ann Surg Oncol. 2015;22(8):2615-2623.

12. U.S. Census Bureau. Census Regions and Divisions of the United States. 2017 https://www2.census.gov/geo/pdfs/maps-data/maps/ reference/us_regdiv.pdf. Accessed 14 Jun 2017.

13. Birkmeyer JD, Dimick JB. Potential benefits of the new Leapfrog standards: effect of process and outcomes measures. Surgery. 2004;135(6):569-575.

14. Metzger R, Bollschweiler E, Vallböhmer D, et al. High volume centers for esophagectomy: what is the number needed to achieve low postoperative mortality?. Dis Esophagus. 2004;17(4): 310-314.

15. Fuchs HF, Harnsberger CR, Broderick RC, et al. Mortality after esophagectomy is heavily impacted by center volume: retrospective analysis of the Nationwide Inpatient Sample. Surg Endosc. 2017;31(6):2491-2497.

16. Birkmeyer JD, Stukel TA, Siewers AE, et al. Surgeon volume and operative mortality in the United States. $N$ Engl J Med. 2003;349(22):2117-2127.

17. Speicher PJ, Englum BR, Ganapathi AM, et al. Traveling to a high-volume center is associated with improved survival for patients with esophageal cancer. Ann Surg. 2017;265(4):743-749. 